\title{
Gyto-Sevis: semantic similarity-based visualisation of protein interaction networks
}

\section{P.H. Guzzi ${ }^{\bowtie}$, M. Cannataro}

Department of Medical and Surgical Sciences, University "'Magna Graecia"” of Catanzaro, Catanzaro, Italy

\section{Motivations}

The analysis of the whole set of molecular interactions in an organism, often referred to as interaction networks, is becoming an important research area. The recent trend in this area is represented by the integration of raw proteomics data with biological knowledge, usually represented as terms and relations into different ontologies (e.g. Gene Ontology). Usually raw proteomics data are enriched by such biological knowledge by annotating them with terms extracted by the ontologies. The use of such annotations for the analysis of protein data is a relatively novel research area that is currently becoming more and more central in research. A main area is represented by the definition of the similarity among genes and proteins on the basis of the annotating terms, also referred to as semantic similarity analysis of protein data. Semantic similarity measure refers to a set of techniques used to evaluate the similarity of two or more terms belonging to the same ontologies. Consequently, they may be used to evaluate the similarity of two genes or proteins starting from the terms extracted from the same ontology used to annotate them. This theoretical framework may be used also for the development of novel visualization techniques for protein interaction networks based on the semantic similarities of proteins [1]. In this scenario three kind of bioinformatics software are appearing: (i) tools for automatic annotation of proteomic data with Gene Ontology terms yielding to annotated protein interaction databases; (ii) tools for querying and filtering in a semantic way such annotated databases; (iii) tools for semantic visualization of annotated protein interaction networks. The work presented here belongs to the third class [2-3].

\section{Methods}

Cyłoscape is a tool for visualizing and analyzing interaction networks, based on an extensible architecture. Cystoscape offers basic visualization techniques where a distinction among nodes (proteins) or edges (interactions) belonging to different classes can be obtained in a manual way by explicitly setting their colors. Moreover, recent Cytoscape plug-ins are involved with Gene Ontology terms and interaction networks. BINGO determines which $\mathrm{GO}$ categories are statistically overrepresented in a set of genes of a sub-graph of a biological network, while GOlorize uses GO categories to guide the network graph layout process and to emphasize the biological function of the nodes. Nevertheless Cytoscape currently does not offer directly or though additional plug-ins, the capabilities to visualize networks in a semantic similarity space.

\section{Results}

CytoSevis is a Cytoscape plugin that is able to visualize protein interaction networks in a semantic similarity space. Based on an intuitive interface it is able to load a network from Cytoscape, to load the semantic similarities provided as a separate file, and to visualize resulting coloured network into Cyłoscape. The main contributions of the proposed plugin are the following: (i) we exploit semantic similarity analysis, that is currently one of the main developing research area in protein interaction networks; (ii) CytoSevis provides a graphical user interface and enables the visualisation of networks in such a space; (iii) we provide a cross-platform Java-based CytoSevis distribution running on Windows/Linux/MacOS; (iv) we provide semantic similarity data on a separate web site. CytoSevis for Windows, Linux and Mac OSX platforms is available under GPL license. User can download it from the Cytoscape web site (http://cytoscape.org). Semantic similarities measures are available on the CytoSevis web site (http://bioingegneria.unicz.it/ guzzi/ss/).

\section{Availability}

http:/cytoscape.org/ 


\section{References}

1. Guzzi P, Mina M. Investigating bias in semantic similarity measures for analysis of protein interactions. In: Proceedings of $1 s t$ International Workshop on Pattern Recognition in Proteomics, Structural Biology and Bioinformatics (PR PS BB 2011) published in Nuovo Cimento. Ravenna, 13th September 2011.
2. Guzzi PH, Milano M, Veltri P, Cannataro M. Semantic similarities as features of protein complexes, accepted in 4 th IWBNA International Workshop on Biomolecular Network Analysis, in conjunction with IEEE BIBM International Conference on Bioinformatics and Biomedicine, Atlanta 11-13 November 2011.

3. Hiram P, Cuzzi M, Mina M, Guerra C, Cannataro M. Semantic Similarity Measures: Assessment with biological features and Issues, to appear in Briefings In Bioinformatics Oxford Journal 10.1093/bib/BBR066 